\title{
On a Coupled Cahn-Hilliard System for Copolymer/Homopolymer Mixtures
}

\author{
Laurence Cherfils ${ }^{1, *}$ and Alain Miranville ${ }^{2}$ \\ ${ }^{1}$ La Rochelle Université, LaSIE, UMR CNRS 7356, Avenue Michel Crépeau, \\ F-17042 La Rochelle Cedex, France; \\ ${ }^{2}$ Laboratoire de Mathématiques et Applications, Université de Poitiers, UMR \\ CNRS 7348, Site du Futuroscope - Téléport 2, 11 Boulevard Marie et Pierre Curie \\ - Bâtiment H3 - TSA 61125, 86073 Poitiers Cedex 9, France.
}

Received July 1, 2020; Accepted October 22, 2020;

Published online January 17, 2022.

\begin{abstract}
Our aim in this paper is to study a coupled Cahn-Hilliard system for copolymer/homopolymer mixtures. We prove the existence, uniqueness and regularity of solutions. We then prove the existence of finite dimensional global attractors.
\end{abstract}

AMS subject classifications: 35K55, 35B45, 35B41

Key words: Coupled Cahn-Hilliard system, well-posedness, finite dimensional global attractors

\section{Introduction}

Our aim in this paper is to study a coupled Cahn-Hillard model for copolymer/homopolymer mixtures.

The original Cahn-Hilliard equation,

$$
\frac{\partial u}{\partial t}+\Delta^{2} u-\Delta f(u)=0,
$$

was initially proposed to model phase separation processes in binary alloys (see [6,7]). Since then, this equation, or some of its variants, were successfully applied to many other applications than just phase separation in alloys. We can mention, for instance, dealloying (this can be observed in corrosion processes; see [14]), population dynamics (see [11]), tumor growth (see [1,15,16, 21, 26]), bacterial films (see [22]), thin films (see [31]), chemistry (see [35]), image processing (see $[5,8,13]$ ) and even astronomy,

*Corresponding author. Email addresses: laurence.cherfils@univ-lr.fr (Cherfils L), Alain.Miranville@math. univ-poitiers.fr (Miranville A) 
with the rings of Saturn (see [33]), and ecology (for instance, the clustering of mussels can be perfectly well described by the Cahn-Hilliard equation; see [24]). We refer the interested reader to $[25,28]$ for reviews on the Cahn-Hilliard equation and some of its variants, as well as their mathematical analysis. The numerical analysis of the Cahn-Hilliard equation was addressed, e.g., in [37, 38, 40, 41] (see also [25] for more references).

Block copolymer materials are important in engineering as they have the ability to create a wide variety of micro-structures resulting from a compromise between phase segregation and polymer architecture which prevents complete phase separation (see, e.g., $[4,12,20])$. In particular, diblock copolymers have been studied from a mathematical and numerical point of view (see, e.g., $[9,17]$ and references therein).

In this paper, we consider a coupled Cahn-Hilliard system considered in $[2,3]$ (see also [10]) to study the phase separation of mixtures consisting of a homopolymer and a copolymer. More precisely, the two phase variables were introduced to describe the macro-phase separation between the homopolymer and copolymer, as well as the micro-phase separation between the two components of the diblock copolymers. The model consists of the coupling of the Ohta-Kawasaki equation,

$$
\frac{\partial u}{\partial t}+\Delta^{2} u+\sigma \bar{u}-\Delta f(u)=0, \quad \sigma>0,
$$

where $\bar{u}$ is the difference between $u$ and its spatial average (see [29]; this equation actually is a variant of the Cahn-Hilliard-Oono equation,

$$
\frac{\partial u}{\partial t}+\Delta^{2} u+\sigma u-\Delta f(u)=0, \quad \sigma>0,
$$

proposed in [30] to model long-ranged effects), describing microscopic phase segregation, and the Cahn-Hillard equation. A related sharp interface model was proposed in [34], where global energy minimizers were studied. More precisely, there, existence and characterization of minimizers, together with upper and lower bounds on their energy, were obtained in one space dimension. In higher space dimensions, one only has upper bounds. Another related three-components model was considered in $[18,19]$, where the existence and stability of equilibria, which are minimizers of the energy, were studied (see also [36] for a similar model with nonlocal interactions).

Efficient numerical simulations for the coupled Cahn-Hilliard model were performed in [23], based on an uncoupled and second-order unconditionally energy stable scheme. More precisely, there, two time marching schemes were proposed and their unique solvability and unconditional energy stability were established.

In this paper, we address the mathematical analysis of the problem. More precisely, we first prove the existence, uniqueness and regularity of solutions. We then address the asymptotic behavior of the associated dynamical system, in terms of finite dimensional global attractors. 


\section{Setting of the problem}

We consider the following initial and boundary value problem in a bounded and regular domain $\Omega \subset \mathbb{R}^{n}, n=1,2$ or 3 , with boundary $\Gamma$ :

$$
\begin{aligned}
& \frac{\partial u}{\partial t}+\Delta^{2} u-\Delta \frac{\partial F}{\partial u}=0, \\
& \frac{\partial v}{\partial t}+\Delta^{2} v-\Delta \frac{\partial F}{\partial v}+\sigma \bar{v}=0, \quad \sigma>0, \\
& \frac{\partial u}{\partial v}=\frac{\partial \Delta u}{\partial v}=\frac{\partial v}{\partial v}=\frac{\partial \Delta v}{\partial v}=0 \quad \text { on } \Gamma, \\
& \left.u\right|_{t=0}=u_{0},\left.\quad v\right|_{t=0}=v_{0} .
\end{aligned}
$$

Here,

$$
\begin{aligned}
& F(u, v)=H(u)+H(v)+\alpha u v+\beta u v^{2}, \quad \alpha, \beta \in \mathbb{R}, \\
& \frac{\partial F}{\partial u}(u, v)=f(u, v)=h(u)+\alpha v+\beta v^{2}, \quad \frac{\partial F}{\partial v}(u, v)=g(u, v)=h(v)+\alpha u+2 \beta u v, \\
& H(s)=\frac{1}{4}\left(s^{2}-1\right)^{2}, \quad h(s)\left(=H^{\prime}(s)\right)=s^{3}-s .
\end{aligned}
$$

Note in particular that

$$
h^{\prime} \geq-1
$$

Furthermore, it follows from Young's inequality that

$$
\begin{array}{ll}
c_{1} s^{4}-c_{2} \leq H(s) \leq c_{3} s^{4}+c_{4}, & c_{1}, c_{3}>0, c_{2}, c_{4} \geq 0, s \in \mathbb{R}, \\
h(s)(s-m) \geq c_{5} s^{4}-c_{6, m}, & c_{5}>0, c_{6, m} \geq 0, s, m \in \mathbb{R},
\end{array}
$$

where $c_{6, m}$ depends continuously on $m$. We also set, for $w \in L^{1}(\Omega)$,

$$
\langle w\rangle=\frac{1}{\operatorname{Vol}(\Omega)} \int_{\Omega} w(x) d x,
$$

and for $w \in H^{-1}(\Omega)$,

$$
\langle w\rangle=\frac{1}{\operatorname{Vol}(\Omega)}\langle w, 1\rangle_{H^{-1}(\Omega), H^{1}(\Omega)} .
$$

We finally set, whenever it makes sense,

$$
\bar{w}=w-\langle w\rangle .
$$


Remark 2.1. We can more generally consider here any regular function $H$ such that $h^{\prime} \geq-c_{0}, c_{0} \geq 0$, and satisfying (2.6)-(2.7). we can also consider any polynomial growth of the form $a s^{2 p+2}, p \in \mathbb{N}, a>0$. We will however give a regularity result which requires $p=1$ when $n=3$.

We denote by $((\cdot, \cdot))$ the usual $L^{2}$-scalar product, with associated norm $\|\cdot\|$. We also set $\|\cdot\|_{-1}=\left\|(-\Delta)^{-\frac{1}{2}} \cdot\right\|$, where $(-\Delta)^{-1}$ denotes the inverse of the minus Laplace operator associated with Neumann boundary conditions and acting on functions with null spatial average. More generally, we denote by $\|\cdot\|_{X}$ the norm on the Banach space $X$.

We note that

$$
\begin{gathered}
w \mapsto\left(\|\bar{w}\|_{-1}^{2}+\langle w\rangle^{2}\right)^{\frac{1}{2}}, \quad w \mapsto\left(\|\bar{w}\|^{2}+\langle w\rangle^{2}\right)^{\frac{1}{2}} \\
w \mapsto\left(\|\nabla w\|^{2}+\langle w\rangle^{2}\right)^{\frac{1}{2}} \text { and } w \mapsto\left(\|\Delta w\|^{2}+\langle w\rangle^{2}\right)^{\frac{1}{2}}
\end{gathered}
$$

are norms on $H^{-1}(\Omega), L^{2}(\Omega), H^{1}(\Omega)$ and $H^{2}(\Omega)$, respectively, which are equivalent to the usual norms on these spaces. Furthermore, $\|\cdot\|_{-1}$ is a norm on $\left\{w \in H^{-1}(\Omega),\langle w\rangle=\right.$ $0\}$ which is equivalent to the usual $H^{-1}$-norm.

Throughout this paper, the same letters $c$ and $c^{\prime}$ denote (generally positive) constants which may vary from line to line, or even in a same line. The same holds for $c_{\delta_{1}, \delta_{2}}, \ldots$ which denotes a constant depending on the parameters $\delta_{1}, \delta_{2}, \cdots$.

\section{Well-posedness}

We have the following result.

Theorem 3.1. We assume that $\left(u_{0}, v_{0}\right) \in H^{1}(\Omega)^{2}$. Then, (2.1)-(2.4) possesses a unique weak solution $(u, v)$ such that, $\forall T>0$,

$$
(u, v) \in L^{\infty}\left(0, T ; H^{1}(\Omega)^{2}\right) \cap L^{2}\left(0, T ; H^{2}(\Omega)^{2}\right)
$$

and

$$
\left(\frac{\partial u}{\partial t}, \frac{\partial v}{\partial t}\right) \in L^{2}\left(0, T ; H^{-1}(\Omega)^{2}\right)
$$

\section{Proof. Existence:}

The proof of existence can be carried out via a standard Galerkin scheme. Here below, we only give formal estimates which can be justified by the aforementioned scheme.

First, note that, integrating (2.1) and (2.2) over $\Omega$ and by parts, we obtain, owing to the boundary conditions (2.3),

$$
\langle u(t)\rangle=\left\langle u_{0}\right\rangle, \quad\langle v(t)\rangle=\left\langle v_{0}\right\rangle, \quad t \geq 0 .
$$


We then rewrite the problem in the following equivalent form:

$$
\begin{aligned}
& (-\Delta)^{-1} \frac{\partial u}{\partial t}-\Delta u+\frac{\overline{\partial F}}{\partial u}=0 \\
& (-\Delta)^{-1} \frac{\partial v}{\partial t}-\Delta v+\frac{\frac{\partial F}{\partial v}}{\partial v}+\sigma(-\Delta)^{-1} \bar{v}=0 \\
& \frac{\partial u}{\partial v}=\frac{\partial v}{\partial v}=0 \quad \text { on } \Gamma \\
& \left.u\right|_{t=0}=u_{0},\left.\quad v\right|_{t=0}=v_{0} .
\end{aligned}
$$

Note indeed that $\left\langle\frac{\partial u}{\partial t}\right\rangle=\left\langle\frac{\partial v}{\partial t}\right\rangle=0$.

We multiply (3.2) by $\frac{\partial u}{\partial t}$ and (3.3) by $\frac{\partial v}{\partial t}$, integrate over $\Omega$ and by parts and sum the two resulting equalities to find

$$
\frac{1}{2} \frac{d}{d t}\left(\|\nabla u\|^{2}+\|\nabla v\|^{2}+\sigma\|\bar{v}\|_{-1}^{2}\right)+\left\|\frac{\partial u}{\partial t}\right\|_{-1}^{2}+\left\|\frac{\partial v}{\partial t}\right\|_{-1}^{2}+\left(\left(\frac{\partial F}{\partial u}, \frac{\partial u}{\partial t}\right)\right)+\left(\left(\frac{\partial F}{\partial v}, \frac{\partial v}{\partial t}\right)\right)=0,
$$

which yields, noting that

$$
\left(\left(\frac{\partial F}{\partial u}, \frac{\partial u}{\partial t}\right)\right)+\left(\left(\frac{\partial F}{\partial v}, \frac{\partial v}{\partial t}\right)\right)=\frac{d}{d t} \int_{\Omega} F(u, v) d x
$$

the energy equality

$$
\frac{d}{d t}\left(\|\nabla u\|^{2}+\|\nabla v\|^{2}+\sigma\|\bar{v}\|_{-1}^{2}+2 \int_{\Omega} F(u, v) d x\right)+2\left(\left\|\frac{\partial u}{\partial t}\right\|_{-1}^{2}+\left\|\frac{\partial v}{\partial t}\right\|_{-1}^{2}\right)=0 .
$$

We set, in what follows, recalling (3.1),

$$
\left\langle u_{0}\right\rangle=\kappa_{1}, \quad\left\langle v_{0}\right\rangle=\kappa_{2}, \quad \kappa_{1}, \kappa_{2} \in \mathbb{R} .
$$

In particular, the constants below may depend on $\kappa_{1}$ and $\kappa_{2}$. We however do not write such a dependence explicitly.

That said, we next multiply (3.2) by $\bar{u}$ and (3.3) by $\bar{v}$ and have, summing the two resulting equalities,

$$
\begin{gathered}
\frac{1}{2} \frac{d}{d t}\left(\|\bar{u}\|_{-1}^{2}+\|\bar{v}\|_{-1}^{2}\right)+\|\nabla u\|^{2}+\|\nabla v\|^{2}+\sigma\|\bar{v}\|_{-1}^{2} \\
+((f(u, v), \bar{u}))+((g(u, v), \bar{v}))=0 .
\end{gathered}
$$

Note that 


$$
\begin{aligned}
& ((f(u, v), \bar{u}))+((g(u, v), \bar{v})) \\
= & ((h(u), \bar{u}))+((h(v), \bar{v}))+2 \alpha((u, v))+3 \beta\left(\left(u, v^{2}\right)\right) \\
& \quad-\alpha \kappa_{1}((v, 1))-\beta \kappa_{1}((v, v))-\alpha \kappa_{2}((u, 1))-2 \beta \kappa_{2}((u, v)) \\
\geq & \frac{c_{5}}{2}\left(\|u\|_{L^{4}(\Omega)}^{4}+\|v\|_{L^{4}(\Omega)}^{4}\right)-c,
\end{aligned}
$$

owing to (2.7) and Young's inequality. It thus follows from (3.7)-(3.8) that

$$
\frac{d}{d t}\left(\|\bar{u}\|_{-1}^{2}+\|\bar{v}\|_{-1}^{2}\right)+\|\nabla u\|^{2}+\|\nabla v\|^{2}+\sigma\|\bar{v}\|_{-1}^{2}+c_{5}\left(\|u\|_{L^{4}(\Omega)}^{4}+\|v\|_{L^{4}(\Omega)}^{4}\right) \leq c .
$$

Summing (3.6) and (3.9), we also deduce from (2.6) the differential inequality

$$
\frac{d E}{d t}+c\left(E+\left\|\frac{\partial u}{\partial t}\right\|_{-1}^{2}+\left\|\frac{\partial v}{\partial t}\right\|_{-1}^{2}\right) \leq c^{\prime}, \quad c>0,
$$

where

$$
E=\|\nabla u\|^{2}+\|\nabla v\|^{2}+\sigma\|\bar{v}\|_{-1}^{2}+2 \int_{\Omega} F(u, v) d x+\|\bar{u}\|_{-1}^{2}+\|\bar{v}\|_{-1}^{2}
$$

satisfies

$$
\begin{aligned}
c\left(\|u\|_{H^{1}(\Omega)}^{2}+\right. & \left.\|v\|_{H^{1}(\Omega)}^{2}+\|u\|_{L^{4}(\Omega)}^{4}+\|v\|_{L^{4}(\Omega)}^{4}\right)-c^{\prime} \leq E \\
& \leq c^{\prime \prime}\left(\|u\|_{H^{1}(\Omega)}^{2}+\|v\|_{H^{1}(\Omega)}^{2}+\|u\|_{L^{4}(\Omega)}^{4}+\|v\|_{L^{4}(\Omega)}^{4}\right)+c^{\prime \prime \prime},
\end{aligned}
$$

for $c, c^{\prime \prime}>0, c^{\prime}, c^{\prime \prime \prime} \geq 0$. Indeed, note that it follows from Young's inequality and (2.6) that

$$
\begin{aligned}
\|u\|_{L^{4}(\Omega)}^{4}+\|v\|_{L^{4}(\Omega)}^{4} & \geq \frac{1}{2}\left(\|u\|_{L^{4}(\Omega)}^{4}+\|v\|_{L^{4}(\Omega)}^{4}\right)+\|u\|^{2}+\|v\|^{2}-c \\
& \geq c \int_{\Omega} F(u, v) d x+\|u\|^{2}+\|v\|^{2}-c^{\prime}, \quad c>0,
\end{aligned}
$$

so that

$$
\|\nabla u\|^{2}+\|\nabla v\|^{2}+\sigma\|\bar{v}\|_{-1}^{2}+c_{5}\left(\|u\|_{L^{4}(\Omega)}^{4}+\|v\|_{L^{4}(\Omega)}^{4}\right) \geq c E-c^{\prime}, c>0 .
$$

We finally multiply (3.2) by $-\Delta u$ and (3.3) by $-\Delta v$ and obtain, summing the two resulting equalities,

$$
\begin{aligned}
& \frac{d}{d t}\left(\|\bar{u}\|^{2}+\|\bar{v}\|^{2}\right)+\|\Delta u\|^{2}+\|\Delta v\|^{2}+\sigma\|\bar{v}\|^{2} \\
& \quad+\left(\left(\frac{\partial f}{\partial u} \nabla u, \nabla u\right)\right)+\left(\left(\left(\frac{\partial f}{\partial v}+\frac{\partial g}{\partial u}\right) \nabla u, \nabla v\right)\right)+\left(\left(\frac{\partial g}{\partial v} \nabla v, \nabla v\right)\right)=0,
\end{aligned}
$$


where

$$
\frac{\partial f}{\partial u}=h^{\prime}(u), \quad \frac{\partial f}{\partial v}=\alpha+2 \beta v, \quad \frac{\partial g}{\partial u}=\alpha+2 \beta v, \quad \frac{\partial g}{\partial v}=h^{\prime}(v)+2 \beta u .
$$

We note that it follows from (2.5) that

$$
\begin{aligned}
& \left(\left(\frac{\partial f}{\partial u} \nabla u, \nabla u\right)\right)+\left(\left(\left(\frac{\partial f}{\partial v}+\frac{\partial g}{\partial u}\right) \nabla u, \nabla v\right)\right)+\left(\left(\frac{\partial g}{\partial v} \nabla v, \nabla v\right)\right) \\
& \quad \geq-c\left(\|\nabla u\|^{2}+\|\nabla v\|^{2}\right)+4 \beta((v \nabla u, \nabla v))+2 \beta((u \nabla v, \nabla v)) .
\end{aligned}
$$

Furthermore, owing to Hölder's inequality and a proper Sobolev embedding,

$$
\begin{aligned}
& |4 \beta((v \nabla u, \nabla v))+2 \beta((u \nabla v, \nabla v))| \\
\leq & c\left(\|v\|_{L^{4}(\Omega)}\|\nabla u\|_{L^{4}(\Omega)}\|\nabla v\|+\|u\|_{L^{4}(\Omega)}\|\nabla v\|_{L^{4}(\Omega)}\|\nabla v\|\right) \\
\leq & c\left(\|v\|_{H^{1}(\Omega)}\|u\|_{H^{2}(\Omega)}\|v\|_{H^{1}(\Omega)}+\|u\|_{H^{1}(\Omega)}\|v\|_{H^{2}(\Omega)}\|v\|_{H^{1}(\Omega)}\right) \\
\leq & \frac{1}{2}\left(\|\Delta u\|^{2}+\|\Delta v\|^{2}\right)+c\left(\|u\|_{H^{1}(\Omega)}^{2}\|v\|_{H^{1}(\Omega)}^{2}+\|v\|_{H^{1}(\Omega)}^{4}+1\right) .
\end{aligned}
$$

It thus follows from (3.11)-(3.13) that

$$
\begin{aligned}
& \frac{d}{d t}\left(\|\bar{u}\|^{2}+\|\bar{v}\|^{2}\right)+c\left(\|u\|_{H^{2}(\Omega)}^{2}+\|v\|_{H^{2}(\Omega)}^{2}\right) \\
& \leq c^{\prime}\left(\|u\|_{H^{1}(\Omega)}^{2}\|v\|_{H^{1}(\Omega)}^{2}+\|v\|_{H^{1}(\Omega)}^{4}+1\right), \quad c>0 .
\end{aligned}
$$

It follows from (3.10) that, for $T>0$ given, $(u, v) \in L^{\infty}\left(0, T ; H^{1}(\Omega)^{2}\right)$ and $\left(\frac{\partial u}{\partial t}, \frac{\partial v}{\partial t}\right) \in$ $L^{2}\left(0, T ; H^{-1}(\Omega)^{2}\right)$. Then, (3.14) yields that $(u, v) \in L^{2}\left(0, T ; H^{2}(\Omega)^{2}\right)$.

\section{Uniqueness:}

Let $\left(u_{1}, v_{1}\right)$ and $\left(u_{2}, v_{2}\right)$ be two solutions with initial data $\left(u_{1,0}, v_{1,0}\right)$ and $\left(u_{2,0}, v_{2,0}\right)$, respectively, such that

$$
\left\langle u_{1,0}\right\rangle=\left\langle u_{2,0}\right\rangle,\left\langle v_{1,0}\right\rangle=\left\langle v_{2,0}\right\rangle .
$$

We have, setting $(u, v)=\left(u_{1}, v_{1}\right)-\left(u_{2}, v_{2}\right)$ and $\left(u_{0}, v_{0}\right)=\left(u_{1,0}, v_{1,0}\right)-\left(u_{2,0}, v_{2,0}\right)$,

$$
\begin{aligned}
& (-\Delta)^{-1} \frac{\partial u}{\partial t}-\Delta u+\overline{f\left(u_{1}, v_{1}\right)-f\left(u_{2}, v_{2}\right)}=0 \\
& (-\Delta)^{-1} \frac{\partial v}{\partial t}-\Delta v+\overline{g\left(u_{1}, v_{1}\right)-g\left(u_{2}, v_{2}\right)}+\sigma(-\Delta)^{-1} \bar{v}=0 \\
& \frac{\partial u}{\partial v}=\frac{\partial v}{\partial v}=0 \quad \text { on } \Gamma \\
& \left.u\right|_{t=0}=u_{0},\left.\quad v\right|_{t=0}=v_{0} .
\end{aligned}
$$


Multiplying (3.15) by $u$ and (3.16) by $v$, we obtain, summing the two resulting equalities and noting that $\langle u\rangle=\langle v\rangle=0$,

$$
\begin{aligned}
& \frac{1}{2} \frac{d}{d t}\left(\|u\|_{-1}^{2}+\|v\|_{-1}^{2}\right)+\|\nabla u\|^{2}+\|\nabla v\|^{2}+\sigma\|v\|_{-1}^{2} \\
& \quad+\left(\left(f\left(u_{1}, v_{1}\right)-f\left(u_{2}, v_{2}\right), u\right)\right)+\left(\left(g\left(u_{1}, v_{1}\right)-g\left(u_{2}, v_{2}\right), v\right)\right)=0
\end{aligned}
$$

Note that, employing (2.5) and Young's inequality,

$$
\begin{gathered}
\quad\left(\left(f\left(u_{1}, v_{1}\right)-f\left(u_{2}, v_{2}\right), u\right)\right)+\left(\left(g\left(u_{1}, v_{1}\right)-g\left(u_{2}, v_{2}\right), v\right)\right)=\left(\left(h\left(u_{1}\right)-h\left(u_{2}\right), u\right)\right) \\
+\left(\left(h\left(v_{1}\right)-h\left(v_{2}\right), v\right)\right)+2 \alpha((u, v))+\beta\left(\left(\left(v_{1}+v_{2}\right) v, u\right)\right)+2 \beta\left(\left(u_{1} v+u v_{2}, v\right)\right) \\
\geq-c\left(\|u\|^{2}+\|v\|^{2}\right)+\beta\left(\left(\left(v_{1}+v_{2}\right) v, u\right)\right)+2 \beta\left(\left(u_{1} v+u v_{2}, v\right)\right) .
\end{gathered}
$$

Furthermore, employing Ladyzhenskaya's (we take $n=3$; the other cases can be dealt with in a similar (even easier) way), Hölder's and Young's inequalities,

$$
\begin{aligned}
\left|\beta\left(\left(\left(v_{1}+v_{2}\right) v, u\right)\right)\right| & \leq c\left(\left\|v_{1}\right\|+\left\|v_{2}\right\|\right)\|u\|_{L^{4}(\Omega)}\|v\|_{L^{4}(\Omega)} \\
& \leq c\left(\left\|v_{1}\right\|+\left\|v_{2}\right\|\right)\|u\|^{\frac{1}{4}}\|\nabla u\|^{\frac{3}{4}}\|v\|^{\frac{1}{4}}\|\nabla v\|^{\frac{3}{4}} \\
& \leq c_{T}\left(\|u\|^{\frac{1}{2}}\|\nabla u\|^{\frac{3}{2}}+\|v\|^{\frac{1}{2}}\|\nabla v\|^{\frac{3}{2}}\right) \\
& \leq \epsilon\left(\|\nabla u\|^{2}+\|\nabla v\|^{2}\right)+c_{\epsilon, T}\left(\|u\|^{2}+\|v\|^{2}\right), \quad \forall \epsilon>0 .
\end{aligned}
$$

Proceeding similarly for the other term, it follows from (3.19)-(3.21) that

$$
\frac{d}{d t}\left(\|u\|_{-1}^{2}+\|v\|_{-1}^{2}\right)+\frac{3}{2}\left(\|\nabla u\|^{2}+\|\nabla v\|^{2}\right) \leq c_{T}\left(\|u\|^{2}+\|v\|^{2}\right) .
$$

Employing finally the interpolation inequality

$$
\|w\|^{2} \leq c\|w\|_{-1}\|\nabla w\|_{,} \quad w \in H^{1}(\Omega), \quad\langle w\rangle=0,
$$

and Young's inequality, we deduce that

$$
\frac{d}{d t}\left(\|u\|_{-1}^{2}+\|v\|_{-1}^{2}\right)+\|\nabla u\|^{2}+\|\nabla v\|^{2} \leq c_{T}\left(\|u\|_{-1}^{2}+\|v\|_{-1}^{2}\right), \quad t \in[0, T] .
$$

In particular, it follows from Gronwall's lemma that

$$
\begin{aligned}
& \left\|u_{1}(t)-u_{2}(t)\right\|_{-1}+\left\|v_{1}(t)-v_{2}(t)\right\|_{-1} \\
\leq & c_{T}\left(\left\|u_{1,0}-u_{2,0}\right\|_{-1}+\left\|v_{1,0}-v_{2,0}\right\|_{-1}\right), \quad t \in[0, T],
\end{aligned}
$$

which yields the continuous dependence with respect to the initial data in the $H^{-1}$ topology, as well as the uniqueness.

We then have the following. 
Theorem 3.2. We further assume that $\left(u_{0}, v_{0}\right) \in H^{2}(\Omega)^{2}$, with $\frac{\partial u_{0}}{\partial v}=\frac{\partial v_{0}}{\partial v}=0$ on $\Gamma$. Then, the solution $(u, v)$ given in Theorem 3.1 satisfies, $\forall T>0$,

$$
(u, v) \in \mathcal{C}\left([0, T] ; H^{2}(\Omega)^{2}\right) \cap L^{2}\left(0, T ; H^{4}(\Omega)^{2}\right)
$$

and

$$
\left(\frac{\partial u}{\partial t}, \frac{\partial v}{\partial t}\right) \in L^{2}\left(0, T ; L^{2}(\Omega)^{2}\right) .
$$

Proof. We multiply (3.2) by $-\Delta^{3} u$ and (3.3) by $-\Delta^{3} v$ and obtain, summing the two resulting equalities,

$$
\begin{gathered}
\frac{1}{2} \frac{d}{d t}\left(\|\Delta u\|^{2}+\|\Delta v\|^{2}\right)+\left\|\Delta^{2} u\right\|^{2}+\left\|\Delta^{2} v\right\|^{2}+\sigma\|\Delta v\|^{2} \\
-\left(\left(\Delta f(u, v), \Delta^{2} u\right)\right)-\left(\left(\Delta g(u, v), \Delta^{2} v\right)\right)=0 .
\end{gathered}
$$

Note that

$$
\begin{aligned}
& \left(\left(\Delta f(u, v), \Delta^{2} u\right)\right) \\
= & \left(\left(\Delta h(u), \Delta^{2} u\right)\right)+\alpha\left(\left(\Delta v, \Delta^{2} u\right)\right)+2 \beta\left(\left(v \Delta v, \Delta^{2} u\right)\right)+2 \beta\left(\left(|\nabla v|^{2}, \Delta^{2} u\right)\right) .
\end{aligned}
$$

It is proved in [25] (we consider the most difficult case $n=3$ ), based on Agmon's and several interpolation inequalities, that

$$
\left|\left(\left(\Delta h(u), \Delta^{2} u\right)\right)\right| \leq \epsilon\left\|\Delta^{2} u\right\|^{2}+c_{\epsilon}\left(1+\|u\|_{H^{1}(\Omega)}^{14}\right) .
$$

Indeed, note that

$$
\left|\left(\left(\Delta h(u), \Delta^{2} u\right)\right)\right| \leq\|\Delta h(u)\|\left\|\Delta^{2} u\right\|
$$

and

$$
\Delta h(u)=h^{\prime}(u) \Delta u+h^{\prime \prime}(u) \nabla u \cdot \nabla u .
$$

Moreover, with our choice of the function $h$, we have $h^{\prime}(s)=3 s^{2}-1$ and $h^{\prime \prime}(s)=6 s$. Therefore,

$$
\|\Delta h(u)\| \leq c\left(\left\|u^{2} \Delta u\right\|+\|\Delta u\|+\|u \nabla u \cdot \nabla u\|\right) .
$$

Employing the interpolation inequality

$$
\|u\|_{H^{2}(\Omega)} \leq c\|u\|_{H^{1}(\Omega)}^{\frac{2}{3}}\|u\|_{H^{4}(\Omega)}^{\frac{1}{3}}
$$

and Agmon's inequality

it follows that

$$
\|u\|_{L^{\infty}(\Omega)} \leq c\|u\|_{H^{1}(\Omega)}^{\frac{1}{2}}\|u\|_{H^{2}(\Omega)^{\prime}}^{\frac{1}{2}}
$$

$$
\|u\|_{L^{\infty}(\Omega)} \leq c\|u\|_{H^{1}(\Omega)}^{\frac{5}{6}}\|u\|_{H^{4}(\Omega)}^{\frac{1}{6}} .
$$

We thus find 


$$
\left\|u^{2} \Delta u\right\| \leq\|u\|_{L^{\infty}(\Omega)}^{2}\|\Delta u\| \leq c\|u\|_{L^{\infty}(\Omega)}^{2}\|u\|_{H^{2}(\Omega)}
$$

so that

$$
\left\|u^{2} \Delta u\right\| \leq c\|u\|_{H^{1}(\Omega)}^{\frac{7}{3}}\|u\|_{H^{4}(\Omega)}^{\frac{2}{3}} .
$$

Next, noting that $H^{4}(\Omega) \subset H^{1}(\Omega)$ with continuous embedding and

$$
\|\Delta u\| \leq c\|u\|_{H^{2}(\Omega)} \leq c\|u\|_{H^{1}(\Omega)}^{\frac{2}{3}}\|u\|_{H^{4}(\Omega)^{\prime}}^{\frac{1}{3}}
$$

it is easy to see that

$$
\|\Delta u\| \leq c\|u\|_{H^{1}(\Omega)}^{\frac{1}{3}}\|u\|_{H^{4}(\Omega)}^{\frac{2}{3}}
$$

We now have

$$
\|u \nabla u \cdot \nabla u\| \leq\|u\|_{L^{\infty}(\Omega)}\|\nabla u\|_{L^{4}(\Omega)}^{2} \leq c\|u\|_{H^{1}(\Omega)}^{\frac{5}{6}}\|u\|_{H^{4}(\Omega)}^{\frac{1}{6}}\|\nabla u\|_{L^{4}(\Omega)}^{2} .
$$

As already mentioned, we concentrate on the most difficult case $n=3$. Note that, in three space dimensions, $H^{\frac{3}{4}}(\Omega) \subset L^{4}(\Omega)$ with continuous embedding, so that

$$
\|\nabla u\|_{L^{4}(\Omega)} \leq c\|u\|_{H^{\frac{7}{4}}(\Omega)} .
$$

Employing the interpolation inequality

$$
\|u\|_{H^{\frac{7}{4}}(\Omega)} \leq c\|u\|_{H^{1}(\Omega)}^{\frac{3}{4}}\|u\|_{H^{4}(\Omega)^{\prime}}^{\frac{1}{4}}
$$

it follows that

$$
\|u \nabla u \cdot \nabla u\| \leq c\|u\|_{H^{1}(\Omega)}^{\frac{5}{6}}\|u\|_{H^{4}(\Omega)}^{\frac{1}{6}}\|u\|_{H^{1}(\Omega)}^{\frac{3}{2}}\|u\|_{H^{4}(\Omega)}^{\frac{1}{2}}
$$

and

$$
\|u \nabla u \cdot \nabla u\| \leq c\|u\|_{H^{1}(\Omega)}^{\frac{7}{3}}\|u\|_{H^{4}(\Omega)}^{\frac{2}{3}} .
$$

Collecting the above estimates, we obtain, employing Young's inequality,

$$
\begin{aligned}
\|\Delta h(u)\| & \leq c\left(\|u\|_{H^{1}(\Omega)}^{\frac{7}{3}}\|u\|_{H^{4}(\Omega)}^{\frac{2}{3}}+\|u\|_{H^{1}(\Omega)}^{\frac{1}{3}}\|u\|_{H^{4}(\Omega)}^{\frac{2}{3}}\right) \\
& \leq c\left(1+\|u\|_{H^{1}(\Omega)}^{\frac{7}{3}}\right)\|u\|_{H^{4}(\Omega)}^{\frac{2}{3}} .
\end{aligned}
$$

We thus deduce, employing again Young's inequality, that

$$
\begin{aligned}
\left|\left(\left(\Delta h(u), \Delta^{2} u\right)\right)\right| & \leq c\left(1+\|u\|_{H^{1}(\Omega)}^{\frac{7}{3}}\right)\|u\|_{H^{4}(\Omega)}^{\frac{2}{3}}\left\|\Delta^{2} u\right\| \\
& \leq c\left(1+\|u\|_{H^{1}(\Omega)}^{\frac{7}{3}}\right)\left(\|u\|_{H^{1}(\Omega)}+\left\|\Delta^{2} u\right\|\right)^{\frac{5}{3}}
\end{aligned}
$$


Employing once more Young's inequality, we find

$$
\left|\left(\left(\Delta h(u), \Delta^{2} u\right)\right)\right| \leq \epsilon\left\|\Delta^{2} u\right\|^{2}+c_{\epsilon}\left(1+\|u\|_{H^{1}(\Omega)}^{14}\right) .
$$

The other terms can be handled in a similar way (and are actually easier to be dealt with, as they are of lower order with respect to $u$ and $v)$. The term $\left(\left(\Delta g(u, v), \Delta^{2} v\right)\right)$ can also be handled in a similar way. We finally end up with the differential inequality

$$
\frac{d}{d t}\left(\|\Delta u\|^{2}+\|\Delta v\|^{2}\right)+\left\|\Delta^{2} u\right\|^{2}+\left\|\Delta^{2} v\right\|^{2} \leq c\left(1+\|u\|_{H^{1}(\Omega)}^{14}+\|v\|_{H^{1}(\Omega)}^{14}\right) .
$$

It follows from (3.28) that $(u, v) \in L^{\infty}\left(0, T ; H^{2}(\Omega)^{2}\right) \cap L^{2}\left(0, T ; H^{4}(\Omega)^{2}\right)$, while the regularity $\left(\frac{\partial u}{\partial t}, \frac{\partial v}{\partial t}\right) \in L^{2}\left(0, T ; L^{2}(\Omega)^{2}\right)$ can be read from (2.1)-(2.2). Finally, employing LionsMagenes's theorem (see, e.g., [25]), we deduce that $(u, v) \in \mathcal{C}\left([0, T] ; H^{2}(\Omega)^{2}\right)$.

Remark 3.1. (i) In one and two space dimensions, we can deal with a polynomial of the form

$$
h(s)=\sum_{i=1}^{2 p+1} a_{i} s^{i}, p \in \mathbb{N}, a_{2 p+1}>0 .
$$

However, the restriction $p=1$ is needed in three space dimensions (see [25]).

(ii) Actually, we can also take $p$ arbitrary in three space dimensions, employing more refined estimates and techniques. We refer the reader to [25] for more details.

\section{Existence of finite dimensional global attractors}

We set

$$
\Phi=\left\{(w, z) \in H^{1}(\Omega)^{2},\langle w\rangle=\kappa_{1},\langle z\rangle=\kappa_{2}\right\}, \kappa_{1}, \kappa_{2} \in \mathbb{R} .
$$

It follows from Theorem 3.1 that we can define the family of continuous (for the $H^{-1}$-topology) solving operators $S(t): \Phi \rightarrow \Phi,\left(u_{0}, v_{0}\right) \mapsto(u(t), v(t)), t \geq 0$. Furthermore, this family of operators forms a semigroup, i.e., $S(0)=I$ and $S(t) \circ S(\tau)=S(t+\tau), t$, $\tau \geq 0$.

Next it follows from (3.10) and Gronwall's lemma that $S(t)$ possesses a bounded absorbing set $\mathcal{B}_{0}$ in $\Phi$, i.e., $\forall B \subset \Phi$ bounded, there exists $t_{0}=t_{0}(B)$ such that $t \geq t_{0}$ implies $S(t) B \subset \mathcal{B}_{0}$. Furthermore, it follows from (3.10) again, (3.28) and the uniform Gronwall's lemma (see, e.g., [32]) that $S(t)$ possesses a bounded absorbing set which is compact in $\Phi$ and bounded in $H^{2}(\Omega)^{2}$. It thus follows from standard results (see, e.g., $[27,32])$ that we have the following.

Theorem 4.1. The semigroup $S(t)$ possesses the global attractor $\mathcal{A}$ such that

(i) $\mathcal{A}$ is compact in $\Phi$ and bounded in $H^{2}(\Omega)^{2}$;

(ii) $\mathcal{A}$ is invariant, $S(t) \mathcal{A}=\mathcal{A}, t \geq 0$; 
(iii) $\mathcal{A}$ attracts the bounded subsets of $\Phi$ in the $H^{-1}$-topology,

$$
\forall B \subset \Phi \text { bounded, } \operatorname{dist}(S(t) B, \mathcal{A}) \rightarrow 0 \text { as } t \rightarrow+\infty,
$$

where dist denotes the Hausdorff semidistance between sets defined as

$$
\operatorname{dist}(A, B)=\sup _{a \in A} \inf _{b \in B}\|a-b\|_{H^{-1}(\Omega)^{2}} .
$$

Remark 4.1. The global attractor is the smallest (for the inclusion) bounded set which attracts the bounded sets of trajectories as time goes to infinity. It can thus be seen as a suitable object in view of the study of the asymptotic behavior of the system; it contains all possible dynamics (see, e.g., [27,32] for more details).

We now recall the following (see [39]).

Proposition 4.1. Let $X$ be a compact subset of the Banach space E. We assume that there exist a Banach space $E_{1}$ such that $E_{1}$ is compactly embedded into $E$ and a mapping $L: X \rightarrow X$ such that $L(X)=X$ and $L$ satisfies the following smoothing property on the difference of two solutions:

$$
\left\|L x_{1}-L x_{2}\right\|_{E_{1}} \leq c\left\|x_{1}-x_{2}\right\|_{E}, \quad \forall x_{1}, x_{2} \in X, c>0 .
$$

Then, the fractal dimension of $X$ is finite (in the topology of $E$ ).

Remark 4.2. The fractal dimension is defined as follows. Let $X \subset E$ be a (relatively) compact set. For $\epsilon>0$, let $N_{\epsilon}(X)$ be the minimal number of balls in $E$ of radius $\epsilon$ which are necessary to cover $X$. Then, the fractal dimension of $X$ is the quantity (which belongs to $[0,+\infty])$

$$
\operatorname{dim}_{\mathrm{F}} X=\limsup _{\epsilon \rightarrow 0^{+}} \frac{\log _{2} N_{\epsilon}(X)}{\log _{2} \frac{1}{\epsilon}}\left(=\limsup _{\epsilon \rightarrow 0^{+}} \frac{\ln N_{\epsilon}(X)}{\ln \frac{1}{\epsilon}}\right) .
$$

We can then prove the following.

Theorem 4.2. The global attractor $\mathcal{A}$ has finite fractal dimension in the $H^{-1}$-topology.

Proof. We again consider two solutions $\left(u_{1}, v_{1}\right)$ and $\left(u_{2}, v_{2}\right)$, now with initial data $\left(u_{1,0}, v_{1,0}\right)$ and $\left(u_{2,0}, v_{2,0}\right)$ belonging to $\mathcal{A}$ and such that

$$
\left\langle u_{1,0}\right\rangle=\left\langle u_{2,0}\right\rangle,\left\langle v_{1,0}\right\rangle=\left\langle v_{2,0}\right\rangle .
$$

Note that it follows from the invariance property that the trajectories are globally (in time) bounded in $H^{2}(\Omega)^{2}$ and, thus, in $L^{\infty}(\Omega)^{2}$.

First, note that, integrating (3.22) over $[0,1]$, it follows from (3.23) (we take here $T=1$ ) that

$$
\int_{0}^{1}\left(\|\nabla u\|^{2}+\|\nabla v\|^{2}\right) d x \leq c\left(\left\|u_{0}\right\|_{-1}^{2}+\left\|v_{0}\right\|_{-1}^{2}\right) .
$$


Next, we multiply (3.15) by $t \frac{\partial u}{\partial t}$ and (3.16) by $t \frac{\partial v}{\partial t}$ and obtain, summing the two resulting equalities,

$$
\begin{aligned}
& \frac{1}{2} \frac{d}{d t}\left(t\|\nabla u\|^{2}+t\|\nabla v\|^{2}+t \sigma\|v\|_{-1}^{2}\right)+t\left\|\frac{\partial u}{\partial t}\right\|_{-1}^{2}+t\left\|\frac{\partial v}{\partial t}\right\|_{-1}^{2} \\
& +t\left(\left(f\left(u_{1}, v_{1}\right)-f\left(u_{2}, v_{2}\right), \frac{\partial u}{\partial t}\right)\right)+t\left(\left(g\left(u_{1}, v_{1}\right)-g\left(u_{2}, v_{2}\right), \frac{\partial v}{\partial t}\right)\right) \\
= & \frac{1}{2}\left(\|\nabla u\|^{2}+\|\nabla v\|^{2}+\sigma\|v\|_{-1}^{2}\right) .
\end{aligned}
$$

Note that

$$
\left|\left(\left(f\left(u_{1}, v_{1}\right)-f\left(u_{2}, v_{2}\right), \frac{\partial u}{\partial t}\right)\right)\right| \leq\left\|\nabla\left(f\left(u_{1}, v_{1}\right)-f\left(u_{2}, v_{2}\right)\right)\right\|\left\|\frac{\partial u}{\partial t}\right\|_{-1}
$$

and

$$
\begin{aligned}
& \quad\left\|\nabla\left(f\left(u_{1}, v_{1}\right)-f\left(u_{2}, v_{2}\right)\right)\right\|=\left\|\nabla\left(\left(u_{1}^{2}+u_{2}^{2}+u_{1} u_{2}-1\right) u+\alpha v+\beta\left(v_{1}+v_{2}\right) v\right)\right\| \\
& =\|\left(2 u_{1} \nabla u_{1}+2 u_{2} \nabla u_{2}+u_{1} \nabla u_{2}+u_{2} \nabla u_{1}\right) u+\left(u_{1}^{2}+u_{2}^{2}+u_{1} u_{2}-1\right) \nabla u \\
& \quad+\alpha \nabla v+\beta\left(\nabla v_{1}+\nabla v_{2}\right) v+\beta\left(v_{1}+v_{2}\right) \nabla v \| \\
& \leq c\left(\left\|u_{1}\right\|_{L^{\infty}(\Omega)}\left\|\nabla u_{1}\right\|_{L^{4}(\Omega)}+\left\|u_{2}\right\|_{L^{\infty}(\Omega)}\left\|\nabla u_{2}\right\|_{L^{4}(\Omega)}+\left\|u_{1}\right\|_{L^{\infty}(\Omega)}\left\|\nabla u_{2}\right\|_{L^{4}(\Omega)}\right. \\
& \left.\quad+\left\|u_{2}\right\|_{L^{\infty}(\Omega)}\left\|\nabla u_{1}\right\|_{L^{4}(\Omega)}\right)\|u\|_{L^{4}(\Omega)} \\
& \quad+\left(\left\|u_{1}\right\|_{L^{\infty}(\Omega)}^{2}+\left\|u_{2}\right\|_{L^{\infty}(\Omega)}^{2}+\left\|u_{1}\right\|_{L^{\infty}(\Omega)}\left\|u_{2}\right\|_{L^{\infty}(\Omega)}+1\right)\|\nabla u\| \\
& \quad+\|\nabla v\|+\left(\left\|\nabla v_{1}\right\|_{L^{4}(\Omega)}+\left\|\nabla v_{2}\right\|_{L^{4}(\Omega)}\right)\|v\|_{L^{4}(\Omega)}+\left(\left\|v_{1}\right\|_{L^{\infty}(\Omega)}+\left\|v_{2}\right\|_{L^{\infty}(\Omega)}\right)\|\nabla v\| \\
& \leq c\left(\left\|u_{1}\right\|_{L^{\infty}(\Omega)}\left\|u_{1}\right\|_{H^{2}(\Omega)}+\left\|u_{2}\right\|_{L^{\infty}(\Omega)}\left\|u_{2}\right\|_{H^{2}(\Omega)}+\left\|u_{1}\right\|_{L^{\infty}(\Omega)}\left\|u_{2}\right\|_{H^{2}(\Omega)}\right. \\
& \left.\quad+\left\|u_{2}\right\|_{L^{\infty}(\Omega)}\left\|u_{1}\right\|_{H^{2}(\Omega)}\right)\|\nabla u\|+\left(\left\|u_{1}\right\|_{L^{\infty}(\Omega)}^{2}+\left\|u_{2}\right\|_{L^{\infty}(\Omega)}^{2}+\left\|u_{1}\right\|_{L^{\infty}(\Omega)}\left\|u_{2}\right\|_{L^{\infty}(\Omega)}+1\right)\|\nabla u\| \\
& \quad+\|\nabla v\|+\left(\left\|v_{1}\right\|_{H^{2}(\Omega)}+\left\|v_{2}\right\|_{H^{2}(\Omega)}\right)\|\nabla v\|+\left(\left\|v_{1}\right\|_{L^{\infty}(\Omega)}+\left\|v_{2}\right\|_{L^{\infty}(\Omega)}\right)\|\nabla v\| \\
& \leq c(\|\nabla u\|+\|\nabla v\|),
\end{aligned}
$$

owing to the continuous embedding $H^{1}(\Omega) \subset L^{4}(\Omega)$ and recalling that $\mathcal{A}$ is bounded in $H^{2}(\Omega)$ and $L^{\infty}(\Omega)$. Proceeding in a similar way for the other term in the left-hand side of (4.2), it follows that

$$
\begin{aligned}
& \frac{1}{2} \frac{d}{d t}\left(t\|\nabla u\|^{2}+t\|\nabla v\|^{2}+t \sigma\|v\|_{-1}^{2}\right)+t\left\|\frac{\partial u}{\partial t}\right\|_{-1}^{2}+t\left\|\frac{\partial v}{\partial t}\right\|_{-1}^{2} \\
\leq & c t(\|\nabla u\|+\|\nabla v\|)\left(\left\|\frac{\partial u}{\partial t}\right\|_{-1}+\left\|\frac{\partial v}{\partial t}\right\|_{-1}\right)+\frac{1}{2}\left(\|\nabla u\|^{2}+\|\nabla v\|^{2}+\sigma\|v\|_{-1}^{2}\right),
\end{aligned}
$$

which yields

$$
\begin{aligned}
& \frac{d}{d t}\left(t\|\nabla u\|^{2}+t\|\nabla v\|^{2}+t \sigma\|v\|_{-1}^{2}\right)+t\left\|\frac{\partial u}{\partial t}\right\|_{-1}^{2}+t\left\|\frac{\partial v}{\partial t}\right\|_{-1}^{2} \\
\leq & c t\left(\|\nabla u\|^{2}+\|\nabla v\|^{2}\right)+\|\nabla u\|^{2}+\|\nabla v\|^{2}+\sigma\|v\|_{-1}^{2} .
\end{aligned}
$$


Employing Gronwall's lemma (over $[0,1])$, we find, in view of (4.1),

$$
\|\nabla u(1)\|^{2}+\|\nabla v(1)\|^{2} \leq c\left(\left\|u_{0}\right\|_{-1}^{2}+\left(\left\|v_{0}\right\|_{-1}^{2}\right) .\right.
$$

We finally deduce from (4.4) that the assumptions of Proposition 4.1 are satisfied, taking $X=\mathcal{A}, L=S(1)$ (recall that $\mathcal{A}$ is invariant), $E=H^{-1}(\Omega)$ and $E_{1}=H^{1}(\Omega)$, which finishes the proof.

Remark 4.3. The finite dimensionality means, roughly speaking, that, even though the phase space has infinite dimension, the reduced dynamics (on the global attractor) can be described, in some proper sense, by a finite number of parameters. Theorem 4.2 thus suggests that the large time behavior of the system is, in some proper sense, finite dimensional. This can, in particular, be useful in view of numerical simulations. Indeed, an upper bound on the fractal dimension of the global attractor, in terms of the physical parameters in the equations, would give an estimate on the number of unknowns which are necessary to capture all possible dynamics.

Remark 4.4. Note that the estimates derived above are uniform with respect to $\sigma$, for, say, $\sigma \in(0,1]$. It would thus be interesting to study the dynamics of the model as $\sigma \rightarrow 0^{+}$. In particular, as far as the Cahn-Hilliard-Oono equation is concerned, the dynamics is close, in some proper sense, to that of the Cahn-Hilliard equation when $\sigma$ is small (see [25]). More precisely, one can construct robust families of exponential attractors (see also [27]). This will be addressed elsewhere.

\section{References}

[1] Aristotelous A C, Karakashian O A, Wise S M. Adaptive, second-order in time, primitivevariable discontinuous Galerkin schemes for a Cahn-Hilliard equation with a mass source. IMA. J. Numer. Anal., 2015, 35: 1167-1198.

[2] Avalos E, Higuchi T, Teramoto T, et al. Frustrated phases under three-dimensional confinement simulated by a set of coupled Cahn-Hilliard equations. Soft Matter, 2016, 12: 5905-5914.

[3] Avalos E, Teramoto T, Komiyama H, et al. Transformation of block copolymer nanoparticles from ellipsoids with striped lamellae into onionlike spheres and dynamical control via coupled Cahn-Hilliard equations. ACS Omega, 2018, 3(1): 1304-1314.

[4] Bates F S, Fredrickson G H. Block copolymers-designer soft materials. Phys. Today, 1999, 52(2): 32.

[5] Bertozzi A, Esedoglu S, Gillette A. Inpainting of binary images using the Cahn-Hilliard equation. IEEE Trans. Imag. Proc., 2007, 16: 285-291.

[6] Cahn J W. On spinodal decomposition. Acta Metall, 1961, 9: 795-801.

[7] Cahn J W, Hilliard J E. Free energy of a nonuniform system I, Interfacial free energy. J. Chem. Phys., 1958, 28: 258-267.

[8] Chalupeckí V. Numerical studies of Cahn-Hilliard equations and applications in image processing, in Proceedings of Czech-Japanese Seminar in Applied Mathematics (August 4-7, 2004). Czech Technical University in Prague, 2004.

[9] Cheng Q, Yang X, Shen J. Efficient and accurate numerical schemes for a hydrodynamically coupled phase field diblock copolymer model. J . Comput. Phys., 2017, 341: $44-60$. 
[10] Choksi R, Ren X. Diblock copolymer/homopolymer blends: derivation of a density functional theory. Phys. D., 2005, 203: 100-119.

[11] Cohen D, Murray J M A generalized diffusion model for growth and dispersion in a population. J. Math. Biol., 1981, 12: 237-248.

[12] Deng R, Liu S, Li J, et al. Mesoporous block copolymer nanoparticles with tailored structures by hydrogen-bonding-assisted self-assembly. Adv. Mater., 2012, 24: 1889-1893.

[13] Dolcetta I C, Vita S F. Area-preserving curve-shortening flows: from phase separation to image processing. Interfaces Free Bound, 2002, 4: 325-343.

[14] Erlebacher J, Aziz M J, Karma A, et al. Evolution of nanoporosity in dealloying. Nature, 2001, 410: 450-453.

[15] Garcke H, Lam K F, Nurnberg R, et al. A multiphase Cahn-Hilliard-Darcy model for tumour growth with necrosis. Math. Models Methods Appl. Sci., 2018, 28: 525-577.

[16] Garcke H., Lam K. F., Sitka E., et al. Cahn-Hilliard-Darcy model for tumour growth with chemotaxis and active transport. Math. Models Methods Appl. Sci., 2016, 26: 1095-1148.

[17] Giorgini A, Grasselli M, Miranville A. The Cahn-Hilliard-Oono equation with singular potential. Math. Models Methods Appl. Sci., 2017, 27: 2485-2510.

[18] Glasner K. Multilayered equilibria in a density functional model of copolymer-solvent mixtures. SIAM J. Math. Anal., 2017, 49: 1593-1620.

[19] Glasner K. Evolution and competition of block copolymer nanoparticles. SIAM J. Appl. Math., 2019, 79: 28-54.

[20] Hamley I W. The Physics of Block Copolymers, Vol 19. Oxford University Press, Oxford, 1998.

[21] Khain E, Sander L M, A generalized Cahn-Hilliard equation for biological applications. Phys. Rev. E., 2008, 77: 051129.

[22] Klapper I, Dockery J. Role of cohesion in the material description of biofilms. Phys. Rev. E., 2006, 74: 031902.

[23] Li Q, Mei L. Efficient, decoupled and unconditionally energy stable numerical schemes for the coupled Cahn-Hilliard system in copolymer/homopolymer mixtures. Computer Phys. Commun., to appear.

[24] Liu Q X, Doelman A, Rottschäfer V., et al. Phase separation explains a new class of self-organized spatial patterns in ecological systems. Proc. Nation Acad. Sci., http://www.pnas.org/cgi/doi/10.1073/pnas.1222339110.

[25] Miranville A. The Cahn-Hilliard Equation: Recent Advances and Applications. CBMSNSF Regional Conference Series in Applied Mathematics 95, Society for Industrial and Applied Mathematics (SIAM), Philadelphia, PA, 2019.

[26] Miranville A, Rocca E, Schimperna G. On the long time behavior of a tumor growth model. J Diff. Eqns., 2019, 267: 2616-2642.

[27] Miranville A, Zelik S. Attractors for dissipative partial differential equations in bounded and unbounded domains, in Handbook of Differential Equations. Evolutionary Partial Differential Equations, Vol 4. Dafermos C M, Pokorny M eds., Elsevier, Amsterdam, 2008: 103-200.

[28] Novick-Cohen A. The Cahn-Hilliard equation. In Handbook of Differential Equations, Evolutionary Partial Differential Equations, Vol. 4. Dafermos C M, Pokorny M. eds., Elsevier, Amsterdam, 2008: 201-228.

[29] Ohta T, Kawasaki K. Equilibrium morphology of block copolymer melts. Macromolecules, 1986, 19: 2621-2632.

[30] Oono Y, Puri S. Computationally efficient modeling of ordering of quenched phases. Phys. Rev. Lett., 1987, 58: 836-839.

[31] Oron A, Davis S H, Bankoff S G. Long-scale evolution of thin liquid films. Rev. Mod. Phys., 
1997, 69 : 931-980.

[32] Temam R. Infinite-dimensional dynamical systems in mechanics and physics. Second edition, Applied Mathematical Sciences, Vol 68, Springer-Verlag, New York,1997.

[33] Tremaine S. On the origin of irregular structure in Saturn's rings. Astron J., 2003, 125: 894901.

[34] Van Gennip Y, Peletier M A. Copolymer-homopolymer blends: global energy minimisation and global energy bounds. Calc. Var. Partial Diff. Eqns., 2008, 33: 75-111.

[35] Verdasca J, Borckmans P, Dewel G. Chemically frozen phase separation in an adsorbed layer. Phys. Review E., 1995, 52: 4616-4619.

[36] Wang C, Ren X, Zhao Y. Bubble assemblies in ternary systems with long range interaction. Preprint.

[37] Yang X., Zhao J. On linear and unconditionally energy stable algorithms for variable mobility Cahn-Hilliard type equation with logarithmic Flory-Huggins potential. Commun. Comput. Phys., 2019, 25: 703-728.

[38] Yang X, Zhao J, Wang Q, et al. Numerical approximations for a three-component CahnHilliard phase-field model based on the invariant energy quadratization method. Math Models Methods Appl. Sci., 2017, 27: 1993-2030.

[39] Zelik S. The attractor for a nonlinear reaction-diffusion system with a supercritical nonlinearity and its dimension. Rend Accad. Naz. Sci. XL. Mem. Mat. Appl., 2000, 24: 1-25.

[40] Zhang J, Yang X. Unconditionally energy stable large time stepping method for the $L^{2}$ gradient flow based ternary phase-field model with precise nonlocal volume conservation. Computer Methods Appl Mech. Engrg., 2020, 361: 112743.

[41] Zhang J, Zhao J, Gong Y. Error analysis of full-discrete invariant energy quadratization schemes for the Cahn-Hilliard type equation. J. Comput. Appl. Math., 2020, 372: 112719. 